\title{
Left ventricular remodeling in rugby is a physiological adaptation to exercise: a pilot study conducted with high-level athletes
}

\author{
Nuno Dias Rato ${ }^{1,2}$ (D) Joanna Richards ${ }^{1}$
}

Received: 19 February 2021 / Accepted: 14 July 2021 / Published online: 11 October 2021

(c) The Author(s) 2021

\begin{abstract}
Purpose Literature examining left ventricular (LV) structural adaptations to combined strength and endurance training is inconsistent. Rugby is a sport that combines these two exercise modalities, both during training and match play. This study aimed to explore differences in LV structure between high-level rugby players and untrained controls. Body composition analysis was performed to determine the most appropriate indexing variable for LV mass (LVM) and understand if increases in LV represent either a training-related physiological adaptation or reflect the groups' anthropometric differences.

Methods A cross-sectional design compared 10 rugby players and 10 untrained age-matched, male controls. Body composition was obtained by bioelectrical impedance. M-mode echocardiographic imaging was performed on the LV from the parasternal long axis view.

Results Significantly greater end-diastolic interventricular septum, LV internal diameter, posterior wall thickness, LVM and $\mathrm{LVM}$ /fat-free mass (FFM) $(p<0.05)$ were found in rugby players compared to age-matched controls. Moreover, Pearson's correlation tests revealed FFM to be the body composition variable with the strongest correlation to LVM $(r=0.775, p<0.001)$. Conclusion The differences in LV structure between groups suggest that the combined endurance and strength training that rugby athletes are subjected to, induce a process of concentric and eccentric enlargement of the LV structure. Furthermore, the association found with FFM, suggests it to be the most appropriate body scaling variable to index to LVM and, thus, should be considered when describing increases in LVM. The present research suggests that increased LVM in the athletes group represents true physiological adaptations to training.
\end{abstract}

Keywords Athlete's heart $\cdot$ Left ventricle $\cdot$ Left ventricular mass $\cdot$ Rugby $\cdot$ Allometric scaling $\cdot$ Cardiac remodeling

\section{Introduction}

The term "Athlete's Heart" has been used to define the electrical, structural and functional myocardial adaptations promoted by frequent intensive physical training [1]. These changes are known to affect the four cardiac chambers [2,3]; however, changes in the left ventricle (LV) are more clearly and consistently described in the literature [3]. When compared to sedentary controls, athletes tend to show greater LV mass (LVM) [4, 5] due to alterations on the loading

Nuno Dias Rato

nunodiasrato@ismai.pt

1 Institute of Sport and Physical Activity Research, University of Bedfordshire, Bedford, UK

2 Research Center in Sport Sciences, Health Sciences and Human Development (CIDESD), University of Maia, Av. Carlos Oliveira Campos, Castêlo da Maia, Portugal conditions of the heart during exercise [6]. These modifications in the LV morphology are known to be highly dependent on the sport's specificities $[1,6,7]$.

Athletes participating in endurance sports have previously been shown to undergo a process of eccentric hypertrophy of the cardiac muscle which is characterized by a greater LV internal diameter (LVID) in diastole [8]. Whereas, in strength-trained athletes, the increased LVM is mainly attributed to increased LV walls thickness, a process denominated concentric enlargement [8]. It is well established that the eccentric hypertrophy in the LV, induced by regular endurance training, is due to a volume overload associated with the elevated cardiac output verified during endurance exercise $[3,9,10]$. On the contrary, there is no consensus regarding the mechanisms promoting LV concentric hypertrophy in strength-trained athletes. Pluim et al. [3] proposed that the heart walls thicken with weight lifting to cope with the exaggerated blood pressure induced by this type of exercise. 
However, Naylor et al. [7] suggested that, in strength-trained athletes, increases in the LV wall thicknesses might be due to a larger body size induced by strength training.

It can be concluded that the literature is clear when showing that different types of exercise induce different patterns of LV hypertrophy. Nevertheless, studies analyzing cardiac remodeling in mixed-trained athletes (characterized by strength and endurance activities) have demonstrated inconsistent findings $[5,11]$.

Rugby is a sport that elicits a variety of physiological responses as a result of repeated high-intensity sprints and a high frequency of contact. It is, therefore, a sport that combines moderate and high endurance components with moderate strength components [12]. To the best of the researcher's knowledge, few studies have compared LV dimensions between rugby players and sedentary controls. Forsythe et al. [13] demonstrated that rugby players engaged in more than 10 weekly hours of structured exercise, presented greater LV diameters, walls thickness and mass compared to inactive controls. Differences between groups were still significant after scaling for body dimensions, suggesting that rugby training induces physiological eccentric and concentric LV enlargement. Mandigout et al. [14] concluded that amateur players ( $3 \mathrm{~h}$ of training per week) had undergone a process of concentric LV enlargement. However, despite absolute LVM being significantly greater in the amateur group of players compared to controls, the difference between trained and untrained subjects was no longer significant when LVM was indexed to body surface area (BSA). This suggests the increased LVM in amateur rugby players mirrors greater body dimensions compared to controls, rather than physiological adaptation to training. However, when elite players (14-16 $\mathrm{h}$ of training per week) were compared to the sedentary participants, the athletes' group demonstrated a process of both eccentric and concentric hypertrophy of the LV. Moreover, LVM in elite players was also significantly greater when indexed to BSA which indicates that a substantial increase in LVM is a physiological adaptation to the training stimulus. Nonetheless, in the above-mentioned studies [13, 14], LVM was indexed to BSA, which, according to George et al. [15], is not the most suitable body dimension to use with athletes because of the difference in body composition between athletes and non-athletic individuals. When LVM is indexed to more appropriate variables as FFM [16], differences in the LVM between rugby players and controls might not be evident.

Considering that rugby players usually present bigger body dimensions compared to the general population [12], indexing LVM to body dimensions when analyzing the cardiac structure of these athletes is important to understand if $\mathrm{LV}$ remodeling results from physiological adaptations to training stimulus or greater body size [15]. Different body size measurements have been proposed to be indexed with
LVM [17]. However, whilst previous research has highlighted FFM as the most appropriate variable when indexing LVM in athletes $[16,18,19]$, research within this area is still limited.

The aim of this study was to compare LV structure between rugby players and healthy age-matched sedentary counterparts. Additionally, body composition analysis was performed to determine the body composition variable that most correlates with LVM and understand if increases in LV represent true physiological adaptation to the training stimulus or simply reflect the groups' anthropometric differences. It was hypothesized that rugby players would have greater LV dimensions compared to untrained controls and the difference between groups in LVM was expected to remain after indexed to body dimensions.

\section{Methods}

\section{Participants}

Twenty healthy male volunteers participated in the current study. A minimum sample size of 18 participants was calculated to be $80 \%$ powerful (G*Power 3.1, Heinrich-HeineUniversität, Dusseldorf, Germany) based on a previous study [5]. The experimental group included 10 high-level rugby players from different positions who play in the RFU Championship League and had been competitively active for, at least, 4 years before the study. Their training regimen during the season consisted of $3 \mathrm{~h}$ of strength and $4.5 \mathrm{~h}$ of rugby training divided into 6 weekly sessions. The deconditioning period was 6 weeks per year. The control group comprised 10 age-matched untrained males who reported to do less than 150 min of moderate to vigorous physical activity (energy expenditure $>3$ METs [20]) per week, during the 4 years prior to the study. Participants from the control group attended the University of Bedfordshire Sports Science Laboratories where the data were recorded. Data for the rugby players group were collected at the Ampthill Rugby Club's facilities. Individuals with heart abnormalities, high blood pressure (over 140/90 mmHg) or smokers were not included in the present study. Participants' details can be seen in Table 1 . The study was approved by the University Research Ethics Committee and all participants provided informed consent prior to participation.

\section{Anthropometric measurements}

Participant's height was measured with a conventional stadiometer to the nearest $0.1 \mathrm{~cm}$ (HAR- 98.602; Holtain, Pembrokeshire, UK). Height measurement was performed with the participants barefoot and facing forwards as tall and straight as possible with their heels together. Height 
Table 1 Participants characteristics

\begin{tabular}{lcrl}
\hline & $\begin{array}{c}\text { Untrained } \\
(n=10)\end{array}$ & $\begin{array}{c}\text { Trained } \\
(n=10)\end{array}$ & $p$ value \\
\hline Age (years) & $23.50 \pm 2.32$ & $23.50 \pm 3.34$ & 0.939 \\
SBP (mmHg) & $120.90 \pm 12.30$ & $134.20 \pm 6.20$ & $0.028^{*}$ \\
DBP (mmHg) & $75.30 \pm 8.34$ & $76.80 \pm 7.35$ & 0.675 \\
Height $(\mathrm{cm})$ & $175.00 \pm 4.97$ & $187.20 \pm 6.40$ & $0.000^{*}$ \\
Body Mass $(\mathrm{kg})$ & $79.11 \pm 18.55$ & $101.37 \pm 9.02$ & $0.003^{*}$ \\
Fat Free Mass $(\mathrm{kg})$ & $63.57 \pm 10.40$ & $84.45 \pm 7.36$ & $0.001^{*}$ \\
Fat Mass $(\mathrm{kg})$ & $15.56 \pm 9.20$ & $16.92 \pm 3.35$ & 0.669 \\
Body surface area $\left(\mathrm{m}^{2}\right)$ & $1.95 \pm 0.21$ & $2.30 \pm 0.14$ & $0.000^{*}$ \\
\hline
\end{tabular}

$S B P$ systolic blood pressure, $D B P$ diastolic blood pressure

${ }^{*} p<0.05$, significant difference between untrained and trained participants

and body mass were used to calculate BSA, with the Mosteller formula [21]. Body composition was assessed via bioelectrical impedance using a body composition weighting scale (BC418MA Segmental Body Composition Analyser; Cranlea, Birmingham, UK). Measurements were performed with the participants barefoot and in light clothes standing on the scales, while holding its grips. Alcohol, caffeine, exercise, or food intake were not allowed in the $8 \mathrm{~h}$ preceding testing.

\section{Blood pressure}

Resting blood pressure was measured with an automatic blood pressure monitor (Omron M5-I; Cranlea, Birmingham, UK). Blood pressure was recorded after the participants being at seated rest for $5 \mathrm{~min}$. The arm was placed and supported on the horizontal plane, level with the heart and measurements were recorded according to standardized guidelines [22].

\section{Echocardiographic measurements}

M-mode echocardiographic studies were performed using a commercially available instrument (Vivid 7; Mius Ltd., Gloucester, UK). Echocardiographic studies were performed following the current clinical standards from the American Society of Echocardiography [23]. Participants were undressed from the waist up and asked to lay in the left lateral decubitus position with an integrated 3-lead ECG attached in a modified Lead II position. A phased array (3S 2.2-5.0 MHz) transducer was placed at the parasternal window (at the point of the mitral valve) allowing the production of a two-dimensional image of the LV on the parasternal long axis. M-mode imaging was performed at the level of the mitral valve leaflets. Five consecutive cardiac cycles were obtained and with measurement of three consecutive cycles performed and averaged [23]. Data for each participant were collected during a single testing session.

\section{Data analysis}

LV end-diastolic internal diameter (LVIDd) was measured as the minimum separation between the LV septal and posterior wall; end-diastolic interventricular septum (IVSd) was defined as the distance between the septal surface of the right ventricle and the leading edge of the LV surface of the septum; LV posterior wall thickness (LVPWd) was measured from the endocardial surface to the epicardial-pericardial interface (see Fig. 1). All these measurements were performed on three consecutive cardiac cycles in end-diastole which was digitalized at the onset of the $Q$ wave [23]. The mean of the three measurements was then determined and the values were used to calculate LV mass using the formula: $\mathrm{LVM}(\mathrm{g})=0.8 \times 1.04 \times[(\mathrm{LVIDd}+\mathrm{IVSTd}+\mathrm{LVP}-$ WTd)3 - (LVIDd)3] + 0.6 [23]. LVM was indexed to FFM, fat mass (FM), body mass, height ${ }^{2.7}$ and BSA. Relative
Fig. 1 Measurements of end-diastolic inter ventricular septum (IVSd), left ventricular end-diastolic internal diameter (LVIDd) and left ventricle end-diastolic posterior wall thickness (LVPWd)

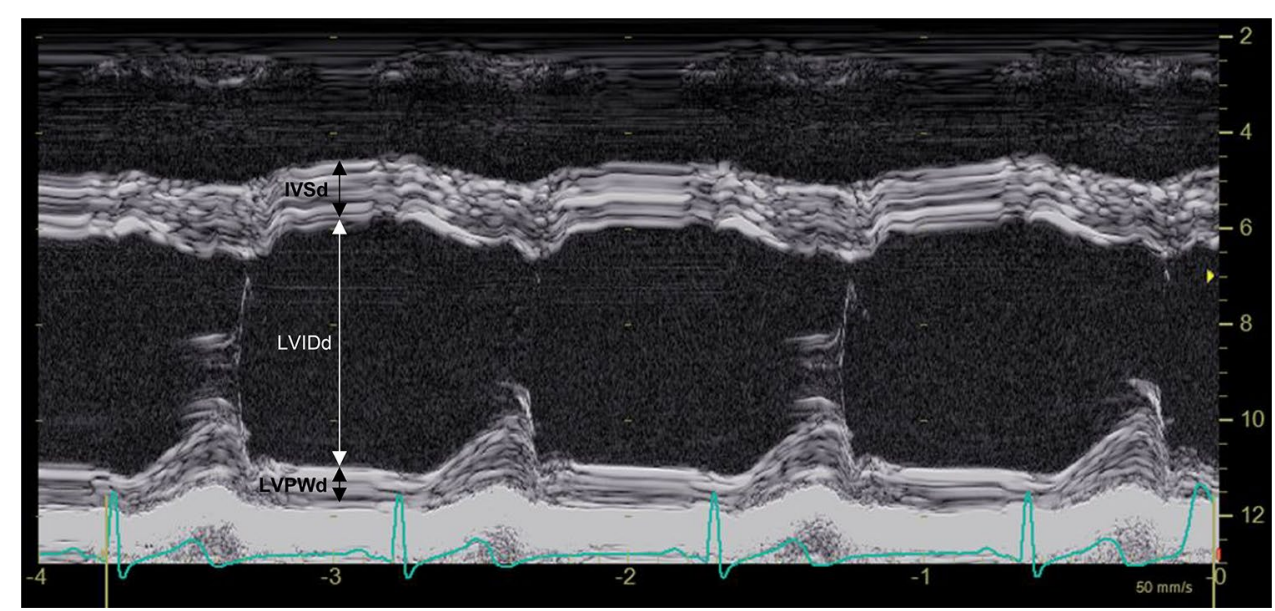


wall thickness (RWT) was calculated using the formula: $\mathrm{RWT}=(2 \times \mathrm{LVPWd}) /(\mathrm{LVIDd})[23]$.

\section{Intra-observer reproducibility}

In a separate group of eleven participants, within-day intraobserver reproducibility was assessed using a coefficient of variation for LV dimensions: IVSd 9.4\%, LVID 2.9\% and PWTd 9.1\%.

\section{Statistical analysis}

Measurements are presented as mean \pm standard deviation. Statistical analyses were performed using the Statistical Package for the Social Sciences for Windows (IBM SPSS statistics version 22, New York, USA). Shapiro-Wilk tests were used to check for normality. All normally distributed variables were analyzed between groups using an independent $T$-test and a non-parametric Mann-Whitney $U$ test was used with non-normally distributed variables. Cohen's $d$ effect sizes were used to examine the magnitude of effect and calculated with a formula proposed by Lakens [24]. Pearson correlation tests were performed to obtain the Pearson correlation coefficient between the body composition measurements and LVM. Significance was set at a $p<0.05$ for all calculations.

\section{Results}

According to the rating scale of $1-4$ proposed by Beaumont et al. [25], 80\% of the echocardiographic images were considered good or excellent.
Control and experimental groups were matched for age $(p>0.05)$. No significant differences between groups were found for diastolic blood pressure $(p>0.05)$ and FM $(p>0.05)$. However, systolic blood pressure, height, body mass, FFM and BSA were significantly greater $(p<0.05)$ in the rugby players (see Table 1 ).

Standard echocardiographic parameters are presented in Table 2. Absolute values of IVSd, LVIDd, LVPWd and LVM were significantly higher in the trained group compared to controls $(p<0.05)$. Once indexed to height, BSA and FFM, LVM was still significantly greater $(p<0.05)$ in the athletes' group compared to controls. Whereas, indexing LVM to FM removed the significant difference in the LVM between groups $(p>0.05)$.

Correlations' graphics can be seen in Fig. 2. FFM was the measure that strongest correlates to LVM $(r=0.775$; $p<0.001)$ followed by BSA $(r=0.737 ; p<0.001)$, weight $(r=0.683 ; p=0.001)$ and height $(r=0.650 ; p=0.002)$. All these variables were positively correlated with LVM. On the contrary, FM was the only body dimension that was shown not to be related to $\operatorname{LVM}(r=0.253 ; p=0.282)$.

\section{Discussion}

The main findings of the current study were that elite rugby players presented greater IVSd, LVIDd, LVPWTd and LVM than controls. Additionally, differences in LVM between groups remained significant after scaling the values for FFM, BSA, height ${ }^{2.7}$ and mass, indicating that rugby training induces physiological concentric and eccentric LV enlargement.

Significant differences in systolic blood pressure were observed between the rugby players and sedentary
Table 2 Echocardiographic LV structure and LVM values indexed to body dimensions

\begin{tabular}{lcccrcrl}
\hline & $\begin{array}{c}\text { Untrained } \\
(n=10)\end{array}$ & $\begin{array}{c}\text { Trained } \\
(n=10)\end{array}$ & $p$ value & \multicolumn{2}{c}{ Confidence Intervals } & Cohen's $d$ \\
\cline { 6 - 7 } & & & & Lower & Upper & \\
\hline IVSd $(\mathrm{cm})$ & $0.86 \pm 0.13$ & $1.31 \pm 0.16$ & $0.000^{*}$ & -0.58 & -0.31 & 3.05 \\
LVIDd $(\mathrm{cm})$ & $4.93 \pm 0.20$ & $5.33 \pm 0.23$ & $0.001^{*}$ & -0.60 & -0.19 & 1.82 \\
LVPWd $(\mathrm{cm})$ & $0.86 \pm 0.11$ & $1.21 \pm 0.15$ & $0.000^{*}$ & -0.47 & -0.23 & 2.72 \\
RWT & $0.35 \pm 0.04$ & $0.46 \pm 0.06$ & $0.001^{*}$ & -0.15 & -0.06 & 2.07 \\
LVM $(\mathrm{g})$ & $151.21 \pm 29.08$ & $283.23 \pm 48.03$ & $0.000^{*}$ & -169.31 & -94.72 & 3.33 \\
LVM/height & $2.7\left(\mathrm{~g} / \mathrm{m}^{2.7}\right)$ & $33.59 \pm 7.39$ & $52.34 \pm 10.09$ & $0.000^{*}$ & -27.06 & -10.44 & 2.12 \\
LVM/body mass $(\mathrm{g} / \mathrm{kg})$ & $1.96 \pm 0.35$ & $2.80 \pm 0.49$ & $0.000^{*}$ & -1.25 & -0.45 & 2.00 \\
LVM/BSA $\left(\mathrm{g} / \mathrm{m}^{2}\right)$ & $77.63 \pm 12.00$ & $123.58 \pm 20.87$ & $0.000^{*}$ & -61.95 & -29.97 & 2.70 \\
LVM/FFM $(\mathrm{g} / \mathrm{kg})$ & $2.40 \pm 0.37$ & $3.37 \pm 0.62$ & $0.000^{*}$ & -1.46 & -0.50 & 1.91 \\
LVM/FM $(\mathrm{g} / \mathrm{kg})$ & $13.24 \pm 7.73$ & $17.13 \pm 3.18$ & 0.158 & -9.45 & 1.66 & 0.66 \\
\hline
\end{tabular}

IVSd interventricular septum in diastole, $L V I D d$ left ventricle internal diameter in diastole, $L V P W d$ left ventricle posterior wall in diastole, $R W T$ relative wall thickness, $L V M$ left ventricle mass, $B S A$ body surface area, FFM fat-free mass, $F M$ fat mass, $N / A$ non-applicable

${ }^{*} p<0.05$, significant difference between untrained and trained participants 
A

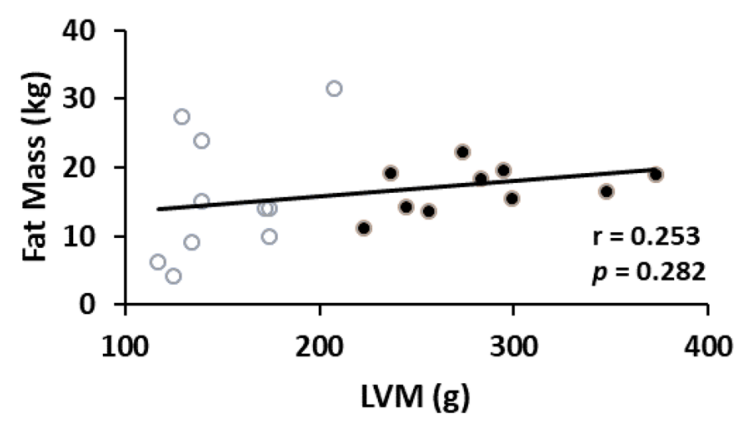

C

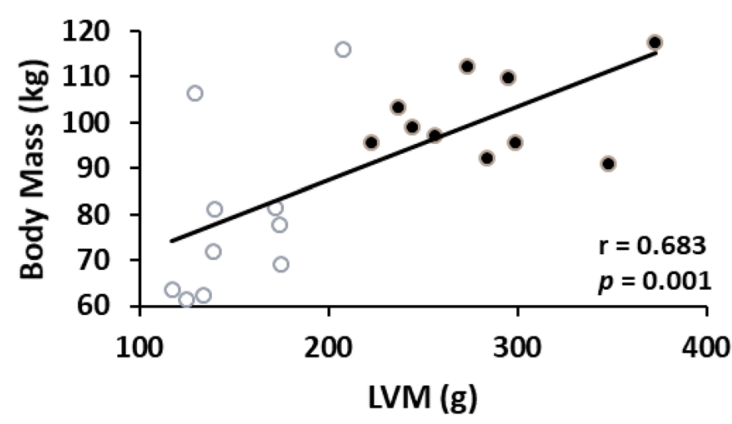

E

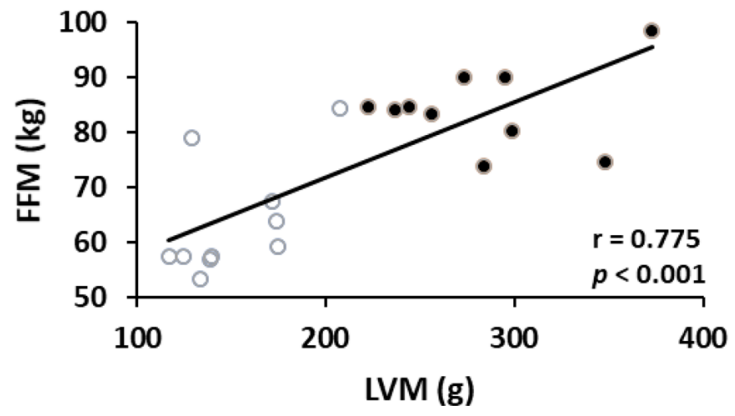

B

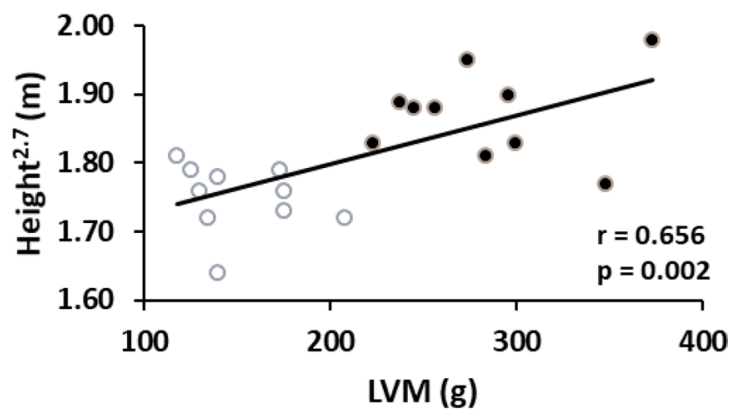

D

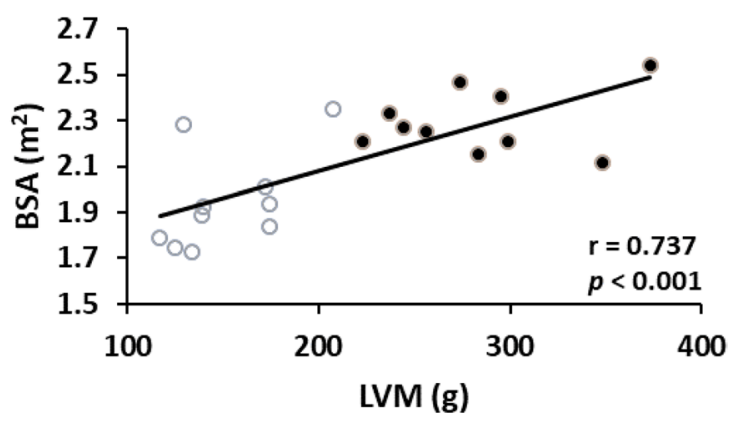

Fig. 2 Pearson correlation tests of left ventricle mass with $\mathbf{A}$ fat mass $\mathbf{B}$ height $\mathbf{C}$ weight $\mathbf{D}$ body surface area (BSA) $\mathbf{E}$ Fat free mass (FFM). Open circles: untrained participants. Solid circles: trained participants

individuals within our sample. Overall, rugby players presented with increased systolic pressure in comparison to controls. This sustained increase in systolic blood pressure within the rugby players could provide an alternative explanation for the observed difference in cardiac structure, as result of 'pressure overload' rather than adaptation to the training stimulus. Grossman et al. [26] have previously found significant increases in wall thickness in pressure overloaded conditions when exploring the pathological heart. However, it should be noted that whilst rugby players presented with increased values in comparison to sedentary individuals, the group mean value of $134 \mathrm{mmHg}$ for systolic blood pressure is not considered hypertensive [22].

Previous research has shown that, when compared to inactive controls, athletes engaged in endurance exercise consistently show significantly increased values of LVIDd and LVM [3]. Existent literature has also reported significantly greater wall thickness in endurance athletes compared to control groups [10,27]. Athletes involved in strength exercise training, however, tend to show significantly greater wall thickness and LVM than inactive controls but similar LVIDd [27, 28]. 
The results of the present study are reinforced by Pluim et al. [3] as rugby players are athletes who are subjected to both endurance and strength training components [12]. Considering the existent literature analyzing LV structure in mixed-trained athletes, Mantziari et al. [5] findings indicate that combined strength and endurance exercise training only promotes concentric LV enlargement in elite rowers, contrasting with the results from the present research. On the contrary, when King et al. [11] assessed the LV structure in elite-rowers, with findings that support the current study, and the theory that mixed-trained athletes undergo a process of both concentric and eccentric enlargement of the LV cavity.

To the best of the researcher's knowledge, only two studies have compared the LV structure between rugby athletes and controls [13, 14]. Forsythe et al. [13] contrasted the LV geometry between rugby players who participated in $>10 \mathrm{~h}$ of structured exercise and non-athletic controls. Concurring with the results from the present research, the authors [13] reported greater absolute and indexed values of LVIDd, walls thickness and LVM in athletes compared to their inactive counterparts. Effect sizes from Forsythe et al. [13] study showed a large effect $(>0.8)$ for LVIDd $(d=1.50)$, $\operatorname{LVM}(d=2.90), \mathrm{LVM} / \mathrm{BSA}(d=1.66)$ and mean wall thickness $(d=1.00)$, which are in line with the large effect sizes observed in the present study. Results from Forsythe et al. [13] support the theory that mixed-type training induces both eccentric and concentric LV enlargement.

Mandigout et al. [14] compared the LV structure of amateur and elite rugby players with controls. When the amateur players were compared to controls, only PWTd and LVM were found to be increased in the athletes' group while no significant differences in LVIDd and IVS were found between groups. However, when LVM was indexed to BSA, differences between groups no longer achieved significance, suggesting that greater LVM in amateur rugby players compared to controls only mirrors bigger body dimensions within the athletes and does not represent true physiological adaptation to training. According to Fagard [2], more than $3 \mathrm{~h}$ of exercise per week is required to promote changes in the LV structure. Considering this group of amateur players only trained $3 \mathrm{~h}$ weekly, this might explain why the amateur athletes did not undergo a process of cardiac remodeling in response to training.

However, when Mandigout et al. [14] compared elite rugby players' $\mathrm{LV}$ dimensions (14-16 h of training per week) with those of the untrained controls, the authors reported similar findings to the current study. Significantly greater values of LVIDd $(d=1.68)$, PWTd $(d=2.09)$, IVSd $(d=1.99), \mathrm{LVM}(d=2.86)$ and LVM/BSA $(d=1.70)$ were reported in the trained group compared to sedentary controls [14]. As LVM was still increased in athletes after considering BSA, suggests that the greater LVM in athletes represents a true physiological adaptation to the exercise stimulus and does not only reflect bigger body size in the athletes' group. Therefore, our results further reinforce those presented by Mandigout et al. [14] with elite players, suggesting that high-level rugby players undergo a process of concentric and eccentric LV enlargement. The large effect sizes observed in Mandigout's study [14] agree with the ones from the current research, additionally strengthening this theory.

It is, however, important to highlight that, according to current standards, rugby players from this study were considered to show LV concentric hypertrophy, as defined by relative wall thickness greater than 0.42 and LVM/BSA greater than $115 \mathrm{~g} / \mathrm{m}^{2}$ [23]. This is reinforced by the greater effect sizes observed for IVSd, PWTd and RWT than LVIDd when untrained controls were compared with the athletes group. This finding contrasts with the previous research presented above $[13,14]$, where rugby athletes demonstrated greater $\mathrm{LV}$ dimensions than controls but were not considered to be in the "hypertrophic" category proposed by Lang et al. [23].

Despite our results agreeing, to some extent, with those reported by Mandiout et al. and Forsythe et al. [13, 14]., they used BSA to index LVM to body size, which may not be the most appropriate variable when indexing LVM to body dimensions, especially in populations where changes in body composition are likely to occur (e.g., athletes) [29]. Another relevant finding from the current study was the difference in the LVM between groups remaining significant after indexing the values to FFM, BSA, height ${ }^{2.7}$ and mass. To account for differences in body size when comparing LV structure between athletes and inactive controls, BSA is often used to index echocardiographic parameters [9, 28]. However, BSA does not take into account differences in body composition $[4,17,15]$. Furthermore, BSA is affected by FM [16], which was not found to be correlated to LVM in the current study. The lack of correlation between FM and LVM might be an explanation for why LVM/FM ratio did not reach a statistically significant difference between groups. Literature has suggested FFM as the most applicable indexing variable to use, especially when studying the cardiac structure in athletes who are expected to have undergone body composition changes $[16,19]$. This is further supported by the present study highlighting FFM as the optimal body dimension for standardization of LVM.

Moreover, Naylor et al. [7] suggested that the increased LV dimensions observed in highly trained athletes might only reflect greater body dimensions in this population and not physiological adaptation to training. Studies performed with endurance [16], and strength-trained [4] athletes have provided support to Naylor's theory by showing that athletes present greater LVM when compared to controls; however, when LVM is indexed to FFM, differences in LVM between groups are no longer significant. In contrast, the present research showed the difference in the LVM between groups was still significant after indexing the values to FFM, BSA, 
height ${ }^{2.7}$ and mass, which suggest that increased LV dimensions in athletes represent a true physiological adaptation to the training stimulus and does not only reflect larger body dimensions (especially lean body mass) that usually characterize athletes when compared to inactive controls.

\section{Limitations}

Despite the relevant findings, the small sample size included in the present study warrants mention. Additionally, when further research compares rugby player's hearts with a sedentary group of controls, we recommend that the players' field position is taken into account as it has been found to have an impact on the LV remodeling [30]. Moreover, in the present study, FFM was determined by bioelectric impedance analysis. Although this method has been validated in the past [31], literature proposes Dual-energy X-ray absorptiometry as the most preferred method for measuring body composition [32]. Nevertheless, as part of the data collection was undertaken offsite, bioelectric impedance analysis was the most efficient and cost-effective solution. It should also be noted that cardiac structure specifically IVSd, LVIDd and PWTd were the main variables examined with the present study. With the effects of myocardial thickening on cardiac function, including longitudinal strain and ejection fraction, currently being inconsistent [33], future research may benefit from exploring measures of cardiac function in addition to the structural measures in this study. Additionally, when interpreting the correlations between LVM and body dimensions that are presented in the current research, it must be acknowledged that, despite correlations between two variables provide information about a pattern in the data, it does not necessarily mean there is a causal relationship between the variables.

\section{Conclusion}

The present study showed increased LVIDd, LVPWTd, IVSd, LVM and LVM/FFM in rugby athletes who were submitted to $7.5 \mathrm{~h}$ of training per week when compared to untrained age-matched controls. These findings indicate that rugby training induces a process of concentric and eccentric enlargement of the LV structure. Although, it is important to mention that, according to the most recent guidelines [14], the athlete's group was only considered to have concentric LV hypertrophy. Moreover, since the LVM was significantly increased in athletes after adjusting the values for body dimensions, our research suggests that the increased dimensions of the LV observed in athletes represent a true physiological adaptation to the training stimulus. In addition to this, FFM was proven to be the body dimension that best correlates with LVM. Therefore, we suggest the ratio LVM/ FFM to be used when comparing the LV structure between athletes and controls with different body sizes.

Author contributions All authors contributed to the study conception and design. Material preparation, data collection and analysis were performed by Nuno Manuel Moreira Dias Rato. The first draft of the manuscript was written by Nuno Manuel Moreira Dias Rato and Joanna Richards and all authors commented on previous versions of the manuscript. All authors read and approved the final manuscript.

\section{Declarations}

Conflict of interest No conflicts of interest, financial or otherwise are declared by the authors.

Ethical approval This study was performed in line with the principles of the Declaration of Helsinki. Approval was granted by the Ethics Committee of the School of Sport Science and Physical Activity of the University of Bedfordshire.

Consent to participate Informed consent was obtained from all participants included in the study.

Open Access This article is licensed under a Creative Commons Attribution 4.0 International License, which permits use, sharing, adaptation, distribution and reproduction in any medium or format, as long as you give appropriate credit to the original author(s) and the source, provide a link to the Creative Commons licence, and indicate if changes were made. The images or other third party material in this article are included in the article's Creative Commons licence, unless indicated otherwise in a credit line to the material. If material is not included in the article's Creative Commons licence and your intended use is not permitted by statutory regulation or exceeds the permitted use, you will need to obtain permission directly from the copyright holder. To view a copy of this licence, visit http://creativecommons.org/licenses/by/4.0/.

\section{References}

1. Maron BJ, Pelliccia A (2006) The heart of trained athletes: cardiac remodeling and the risks of sports, including sudden death. Circulation 114(15):1633-1644

2. Fagard R (2003) Athlete's heart. Heart 89(12):1455-1461

3. Pluim BM, Zwinderman AH, van der Laarse A, van der Wall EE (2000) The athlete's heart: a meta-analysis of cardiac structure and function. Circulation 101(3):336-344

4. George KP, Batterham AM, Jones B (1998) Echocardiographic evidence of concentric left ventricular enlargement in female weightlifters. Eur J Appl Physiol Occup Physiol 79(1):88-92

5. Mantziari A et al (2010) Left ventricular function in elite rowers in relation to training-induced structural myocardial adaptation. Scand J Med Sci Sports 20(3):428-433

6. Fagard R (1996) Athlete's heart: a meta-analysis of the echocardiographic experience. Int J Sports Med 17(3):140-144

7. Naylor LH, George K, O'Driscoll G, Green DJ (2008) The athlete's heart. Sports Med 38(1):69-90

8. Utomi V et al (2013) Systematic review and meta-analysis of training mode, imaging modality and body size influences on 
the morphology and function of the male athlete's heart. Heart 99(23): 1727-1733

9. Arrese AL, Carretero MG, Blasco IL (2006) Adaptation of left ventricular morphology to long-term training in sprint and endurance trained elite runners. Eur J Appl Physiol 96(6):740-746

10. Fagard R, Aubert A, Staessen J, Eynde EV, Vanhees L, Amery A (1984) Cardiac structure and function in cyclists and runners, comparative echocardiographic study. Heart 52(2):124-129

11. King $\mathrm{G}$ et al (2006) Myocardial stiffness and the timing difference between tissue Doppler imaging Ea and peak mitral valve opening can distinguish physiological hypertrophy in athletes from hypertrophic cardiomyopathy. Eur J Echocardiogr 7(6):423-429

12. Gabbett T, King T, Jenkins D (2008) Applied physiology of rugby league. Sports Med 38(2):119-138

13. Forsythe $\mathrm{L}$ et al (2018) The relationship between left ventricular structure and function in the elite rugby football league athlete as determined by conventional echocardiography and myocardial strain imaging. Int J Cardiol 261:211-217

14. Mandigout S, Maufrais C, Cornette T, Alaphilippe A, Daviet J (2016) Speckle tracking echocardiographic analysis of left ventricular systolic and diastolic function in young elite rugby players. J Sports Med Phys Fit 56(11):1401-1409

15. George KP, Batterham AM, Jones B (1998) The impact of scalar variable and process on athlete-control comparisons of cardiac dimensions. Med Sci Sports Exerc 30(6):824-830

16. Whalley GA et al (2004) Association of fat-free mass and training status with left ventricular size and mass in endurance-trained athletes. J Am Coll Cardiol 44(4):892-896

17. de Simone G et al (1992) Left ventricular mass and body size in normotensive children and adults: assessment of allometric relations and impact of overweight. J Am Coll Cardiol 20(5):1251-1260

18. Daniels SR, Loggie JM, Khoury P, Kimball TR (1998) Left ventricular geometry and severe left ventricular hypertrophy in children and adolescents with essential hypertension. Circulation 97(19):1907-1911

19. Hense $\mathrm{H}$ et al (1998) The associations of body size and body composition with left ventricular mass: impacts for indexation in adults. J Am Coll Cardiol 32(2):451-457

20. Norton K, Norton L, Sadgrove D (2010) Position statement on physical activity and exercise intensity terminology. J Sci Med Sport 13(5):496-502

21. Mosteller RD (1987) Simplified calculation of body surface area. N Engl J Med 317(9):1098-1098

22. Williams B et al (2018) 2018 ESC/ESH Guidelines for the management of arterial hypertension: The Task Force for the management of arterial hypertension of the European Society of Cardiology (ESC) and the European Society of Hypertension (ESH). Eur Heart J 39(33):3021-3104
23. Lang RM et al (2015) Recommendations for cardiac chamber quantification by echocardiography in adults: an update from the American Society of Echocardiography and the European Association of Cardiovascular Imaging. J Am Soc Echocardiogr 28(1):234-270

24. Lakens D (2013) Calculating and reporting effect sizes to facilitate cumulative science: a practical primer for t-tests and ANOVAs. Front Psychol 4(863):1-12

25. Beaumont A, Hough J, Sculthorpe N, Richards J (2017) Left ventricular twist mechanics during incremental cycling and knee extension exercise in healthy men. Eur J Appl Physiol 117(1):139-150

26. Grossman W, Jones D, McLaurin LP (1975) Wall stress and patterns of hypertrophy in the human left ventricle. J Clin Investig 56(1):56-64

27. Vinereanu D, Florescu N, Sculthorpe N, Tweddel AC, Stephens MR, Fraser AG (2002) Left ventricular long-axis diastolic function is augmented in the hearts of endurance-trained compared with strength-trained athletes. Clin Sci 103(3):249-257

28. D'Andrea A et al (2007) Biventricular myocardial adaptation to different training protocols in competitive master athletes. Int $\mathbf{J}$ Cardiol 115(3):342-349

29. Chantler PD, Clements RE, Sharp L, George KP, Tan L, Goldspink DF (2005) The influence of body size on measurements of overall cardiac function. Am J Physiol-Heart Circ Physiol 289(5):2059-2065

30. Chevalier L et al (2013) Athlete's heart patterns in elite rugby players: effects of training specificities. Arch Cardiovasc Dis 106(2):72-78

31. Yang SW, Kim TH, Choi HM (2018) The reproducibility and validity verification for body composition measuring devices using bioelectrical impedance analysis in Korean adults. J Exerc Rehabil 14(4):621

32. Shepherd JA, Bennett KNG, Sommer MJ, Heymsfield SB (2017) Body composition by DXA. Bone 104:101-105

33. Haykowsky MJ, Liang Y, Pechter D, Jones LW, McAlister FA, Clark AM (2007) A meta-analysis of the effect of exercise training on left ventricular remodeling in heart failure patients: the benefit depends on the type of training performed. J Am Coll Cardiol 49(24):2329-2336

Publisher's Note Springer Nature remains neutral with regard to jurisdictional claims in published maps and institutional affiliations. 